\title{
Imaging diagnosis of hepatocellular carcinoma: LI-RADS
}

\author{
Guilherme Moura Cunha, Claude B. Sirlin, Kathryn J. Fowler \\ Liver Imaging Group, Department of Radiology, University of California San Diego, San Diego, CA, USA \\ Contributions: (I) Conception and design: GM Cunha, CB Sirlin, KJ Fowler; (II) Administrative support: None; (III) Provision of study materials or \\ patients: None; (IV) Collection and assembly of data: None; (V) Data analysis and interpretation: None; (VI) Manuscript writing: All authors; (VII) \\ Final approval of manuscript: All authors. \\ Correspondence to: Guilherme Moura Cunha; Kathryn J. Fowler. Liver Imaging Group, Department of Radiology, University of California San Diego, \\ 9500 Gilman Dr. San Diego, CA 92093, USA. Email: gcunha@health.ucsd.edu; k1fowler@health.ucsd.edu.
}

\begin{abstract}
Liver cancer is the third most common cause of cancer related death worldwide, $90 \%$ being hepatocellular carcinoma (HCC) and about half of all HCCs estimated to occur in China. Imaging plays a pivotal role in the management of HCC. When stringent criteria are applied to at-risk populations, it enables HCCs to be diagnosed by imaging alone without further need of invasive histology confirmation. To optimize HCC imaging diagnosis and reporting, several systems have been proposed. The Liver Imaging Reporting and Data System (LI-RADS ${ }^{\circledR}$ ) is currently the most comprehensive of these systems, providing guidance on all imaging-related aspects of HCC, from technique for acquisition, reporting, assessment of treatment response and management. For diagnosis, LI-RADS uses major and ancillary imaging features to assign hierarchical categories that communicate the relative probability of HCC to focal liver observations detected in patients at risk. Two LI-RADS algorithms yield high specificity and positive predictive value for HCC diagnosis on contrast enhanced ultrasound (CEUS), CT and MRI. The standardized lexicon and interpretation provided by LI-RADS also improve inter-reader agreement for imaging features and lesion categorization. Additionally, a LI-RADS treatment response algorithm (LR-TR) provide imaging criteria for assessment of response to locoregional therapy. LI-RADS is designed for universal adoption and in this review, we highlighted the most relevant aspects of LI-RADS for the diagnosis of HCC in clinical practice and discussed areas where LI-RADS and Asian guidelines are different.
\end{abstract}

Keywords: Liver; hepatocellular carcinoma (HCC); magnetic resonance imaging (MRI); tomography; ultrasonography

Submitted Feb 28, 2020. Accepted for publication May 14, 2020.

doi: $10.21037 /$ cco-20-107

View this article at: http://dx.doi.org/10.21037/cco-20-107

\section{Introduction}

Liver cancer is the third most common cause of cancer related death worldwide (1). Hepatocellular carcinoma (HCC) comprises $90 \%$ of primary liver malignancies and about half of all HCC cases are estimated to occur in China alone $(2,3)$. Most HCCs develop in patients with chronic liver disease with geographical variability in incidence related to differences in risk factor exposure, with chronic hepatitis $\mathrm{B}$ virus being dominant in southeast Asia and cirrhosis related to hepatitis $\mathrm{C}$ virus, alcohol consumption, or obesity dominating in the West.
Imaging plays an important role in the management of HCC. The specificity of imaging studies for the diagnosis of HCC can be close to $100 \%$, placing HCC amongst few malignancies for which management can be defined based on imaging alone, with no further need for histologic confirmation. This reliance on imaging is currently reflected in multiple medical guidelines (4). Ultrasonography (US) is the method of choice for HCC screening purposes, while contrast-enhanced ultra-sound (CEUS), multiphase computer tomography (CT) and multiphase magnetic resonance imaging (MRI) are recommended for HCC diagnosis (3-7). For diagnosis, imaging 
techniques exploit the vascular alterations that occur during hepatocarcinogenesis. In brief, there is increased arterial supply and reduced portal inflow to malignant nodules, alterations which contribute to the classic arterial phase hyperenhancement and washout appearances on contrast-enhanced multiphase imaging. To optimize the diagnostic accuracy of imaging and standardize the reporting for HCC, several systems have been proposed (8). The Liver Imaging Reporting and Data System (LI-RADS ${ }^{\circledR}$ ) is currently the most comprehensive of these. LI-RADS ${ }^{\circledR}$ aims to standardize and provide guidance on all aspects of HCC imaging, from technique for imaging acquisition to assessing treatment response and directing management.

Currently, LI-RADS provides four algorithms addressing screening and surveillance, diagnosis on CT/ MRI, diagnosis on CEUS, and treatment response on CT/MRI. US LI-RADS, i.e., screening and surveillance LI-RADS, is designed to standardize the reporting of screening and surveillance studies and currently includes guidance for acquisition, interpretation, and reporting of ultrasound exams. Diagnostic CEUS LI-RADS and CT/ MRI LI-RADS strive for high specificity and positive predictive value (PPV) for the imaging diagnosis of HCC. CT/MRI LI-RADS also encompasses staging (radiologic T stage) after diagnosis. Finally, the treatment response algorithm (TR LI-RADS) is designed to assess response to locoregional therapies. Each algorithm has a core document that contains relevant information to be applied in daily clinical practice. A more comprehensive document is also available (LI-RADS manual), which addresses in detail multiple aspects of liver diseases, including imaging parameters, reporting instructions, templates and management recommendations. Most recently, LI-RADS has published a standardized lexicon, which is intended to provide a universal language for liver imaging. All LIRADS materials can be accessed for free at the American College of Radiology website (https://www.acr.org/ClinicalResources/Reporting-and-Data-Systems/LI-RADS).

In this review we provide a summary of the most relevant aspects of LI-RADS for the diagnosis of HCC; as appropriate, we compare and contrast to corresponding Asian guidelines.

\section{The philosophy of diagnostic criteria}

LI-RADS aims for high specificity and PPV to avoid falsepositive examinations in the context of liver transplantation, reducing the risk of erroneous organ allocation. Liver transplantation is considered the best curative option for cirrhotic patients with early stage HCC in the United States. In other regions, such as Asia, where chronic Hepatitis B virus frequently leads to HCC in the absence of cirrhosis or with well compensated cirrhosis, surgical resection may be the best option. Hence in these regions, imaging diagnostic algorithms more suitably focus on sensitivity rather than specificity.

In any scenario, the primary philosophy behind all diagnostic systems is to standardize the approach. The standardized lexicon and interpretation provided by LIRADS have been shown to improve inter-reader agreement for imaging features and lesion categorization (9-13). Furthermore, a standardized approach may lead to better accuracy. Zhang et al. have compared the specificity for the imaging diagnosis of focal liver lesions in patients at risk when using CT/MRI LI-RADS vs. a criteria-free Likert scale. In that study, a significant higher specificity was achieved when the standardized LI-RADS criteria was utilized $(97.3 \%$ vs. $71.2 \%, \mathrm{P}<0.0001)(14)$.

\section{"At risk population" in the United States and around the world}

To ensure a high positive predictive value for the imaging diagnosis of HCC, LI-RADS should be applied only to populations with a high pre-test probability of disease. This "at-risk population" is defined by LI-RADS as adult patients ( $\geq 18$-year-old) with: chronic hepatitis B viral infection even in the absence of liver cirrhosis, liver cirrhosis (with the exception of vascular causes of cirrhosis, see below), or current or prior HCC. By comparison, the Asian-Pacific Association of Study of the Liver (APASL) guidelines allow the imaging-based diagnosis of HCC in the same clinical context, while Japanese, Korean and Chinese guidelines also include patients with chronic hepatitis $\mathrm{C}$ viral infection even in the absence of cirrhosis (3,5-7). LI-RADS does not apply to patients with vascular causes of liver cirrhosis (e.g., cardiac hepatopathy, Budd-Chiari syndrome), because these conditions are associated with hypervascular benign liver lesions, which increase the risk of false positives and reduce the PPV for the diagnosis of HCC.

Determining if a patient meets the at-risk criteria is not always straight forward. The presence of cirrhosis is usually based on clinical context and indicators of advanced liver disease with or without histological confirmation. Although methods such as transient elastography (TE), ultrasound shear wave elastography (SWE), and MR elastography (MRE) may aid in the diagnosis, the results of 
LR-NC Not categorizable (due to image omission or degradation)

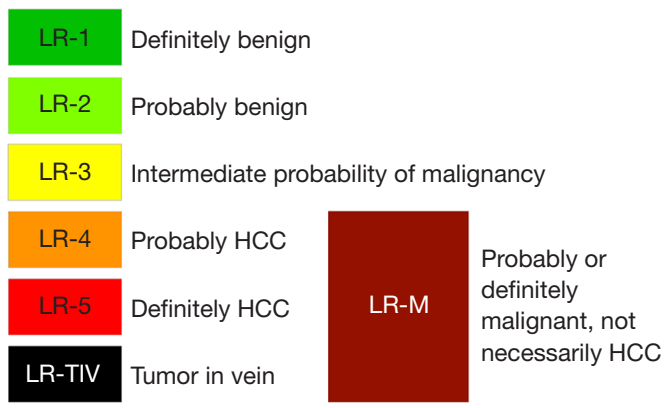

Figure 1 LI-RADS categories communicate the relative probability of overall malignancies and HCC. The LR-NC category denotes observations that cannot be adequately categorized due to technical limitations or artifacts and should be reported with additional workup suggestions [adapted from (20)]. HCC, hepatocellular carcinoma.

these techniques must be incorporated with clinical data to establish a diagnosis of cirrhosis. Emerging evidence from Asia suggests that these techniques might be useful for risk stratification of HCC (15-17), however, further work is needed to establish the role of elastography in determining whether patients meet LI-RADS at-risk criteria in the absence of biopsy or other confirmation of cirrhosis. If the patient is suspected of being at risk for HCC, LI-RADS may be provisionally applied with a suggestion that the referring clinician confirm the patient's risk status.

Another point of controversy is the increasing population of patients with chronic liver disease who do not meet the stringent criteria required by LI-RADS, e.g., patients with advanced fibrosis but not cirrhosis or patients with non-alcoholic fatty liver disease/steatohepatitis (NAFLD/ $\mathrm{NASH}$ ). NAFLD/NASH is currently the leading cause of chronic liver disease world-wide, with the prevalence rising rapidly in Asia (18). Although HCC may develop in patients with NASH even in the absence of cirrhosis (19), there is insufficient evidence to support that LI-RADS would provide a sufficiently high PPV for HCC in this sub-cirrhotic population. Future versions of LI-RADS are expected to address this issue.

\section{Image features and categorization}

In LI-RADS, major and ancillary imaging features of HCC are taken into consideration to assign, in a hierarchical fashion, categories to focal liver observations that communicate the relative probability of HCC. Figure 1 demonstrates the LI-RADS diagnostic categories. The LR-5 category indicates definite HCC. For CT and MRI, the LI-RADS major features include size, presence of nonrim arterial phase hyperenhancement, non-peripheral washout appearance and enhancing capsule appearance. For CEUS, LI-RADS major features are size, non-rim arterial phase hyperenhancement, and late and mild washout. Ancillary features (AFs) may be applied at the radiologist's discretion to adjust the category and to increase confidence in the final diagnosis. AFs may favor malignancy in general, HCC or benignity. Although a larger number of AFs are applied to MRI, they also have been shown to improve categorization of observations on CT (10). Importantly, AFs provide only limited specificity for the diagnosis of HCC, and therefore, cannot be used to diagnose observations that do not otherwise meet stringent criteria for HCC (LR-5). In our practice, these features prove most important for differentiating between LR-3 and LR-4. Table 1 lists LIRADS major and ancillary features.

The major features and criteria for definite HCC vary slightly around the world. In some Asian societal guidelines, the presence of arterial phase hyperenhancement in association with portal venous or delayed phase washout and/or an enhancing capsule allow the definite diagnosis of HCC independently of the observation size, while in LIRADS observations less than $10 \mathrm{~mm}$ in diameter cannot be categorized as LR-5. This restriction of LI-RADS is based on data showing that the diagnostic performance of imaging is reduced for small lesions, leading to insufficiently low PPV (9,21). Similarly, some differences are noted in the definition of washout as a major feature of HCC with hepatobiliary contrast agents (HBA). In LI-RADS and in the APASL guidelines, hypointensity in the portal venous phase only qualifies as washout $(3,20)$. In other Asian societal guidelines, hypointensity in the transitional and/or hepatobiliary phases also qualifies as washout and allows for the diagnosis of HCC (5-7). The reasoning behind this LIRADS recommendation is further described in this text.

\section{Choice of imaging modality: CT, MRI or CEUS}

The choice of imaging modality should consider the clinical scenario, technical advantages and limitations, along with local practices and geographic recommendations. CT or MRI are the most common modalities used in the United 
Table 1 CT/MRI LI-RADS major and ancillary features

Major features
Size
Non-rim Arterial Phase Hyperenhancement*
Washout ${ }^{\star}$
Enhancing Capsule
Threshold growth
Ancillary features

Favoring malignancy in general

US visibility as discrete nodule

Subthreshold growth

Restricted diffusion

Mild-moderate T2 hyperintensity

Corona enhancement

Fat sparing in solid mass

Iron sparing in solid mass

Transitional phase hypointensity

Hepatobiliary phase hypointensity

Favoring $\mathrm{HCC}$ in particular

Nonenhancing "capsule"

Nodule-in-nodule*

Mosaic architecture*

Blood products in mass

Fat in mass, more than adjacent liver

Ancillary features favoring benignity

Size stability $>2$ years*

Size reduction*

Parallels blood pool

Undistorted vessels

Iron in mass, more than liver

Hepatobiliary phase isointensity

Marked T2 hyperintensity

*, also applies to CEUS. MRI, magnetic resonance imaging; $\mathrm{HCC}$, hepatocellular carcinoma.

States, with the widest availability and most user experience. CEUS is often reserved for problem solving. CEUS has high sensitivity to detect hypervascularization, with studies using
LI-RADS criteria showing close to $100 \%$ specificity for the differentiation of HCC and other malignancies $(22,23)$. Currently, the Asian-pacific, Japanese and Chinese guideless recommend CEUS for the diagnosis of $\operatorname{HCC}(3,5,6)$. However, due to the often-limited sonic window and fast contrast kinetics, CEUS is not recommended for evaluation of the whole liver or simultaneous assessment of multiple lesions. In this case, for a more comprehensive assessment and for staging purposes, CT and MRI are the preferred imaging modalities. Even though both diagnostic algorithms (CT/MRI LI-RADS and CEUS LI-RADS) provide high specificity and PPV for the imaging diagnosis of HCC, it is important to understand that the algorithms are not identical as intermodality differences in imaging features exist. Below we summarize each diagnostic algorithm.

\section{Diagnostic algorithms}

Both CT/MRI and CEUS LI-RADS diagnostic algorithms follow a stepwise decision tree for the characterization of focal liver observations. First, readers asses if the examination has limitations or omissions that preclude categorization. If this is the case, observations are assigned a non-categorizable category (LR-NC). Otherwise, the possibility of malignant vascular invasion (LR-TIV), the assessment of benign observations (LR-1, LR-2) or the possibility of malignancies other than HCC (LR-M) are evaluated. Finally, for remaining observations, the probability of $\mathrm{HCC}$ is determined by considering the combination of major features in the diagnostic table. Figure 2 illustrates the LI-RADS diagnostic algorithms and diagnostic tables for CT/MRI and CEUS.

\section{CEUS LI-RADS}

CEUS LI-RADS is designed for use with blood pool microbubble agents that allow the assessment of vascularization and characterization of focal liver observations. Of note, Sonazoid ${ }^{\mathrm{TM}}$ (perfluorobutane, GE Healthcare, Oslo, Norway), a microbubble agent that has prolonged uptake due to higher stability and Kuppfer cells phagocytosis and is available in some Asian countries, has not been adopted by CEUS LI-RADS in v2018 as it is not currently approved for clinical use in the United States (24).

For CEUS LI-RADS, continuous imaging should be performed from contrast injection until or beyond arterial phase peak enhancement to assess hypervascularization and early washout. After 60 seconds of the injection, imaging 
CEUS LI-RADS ${ }^{\circledR}$

\section{CT/MRI LI-RADS ${ }^{\circledR}$}

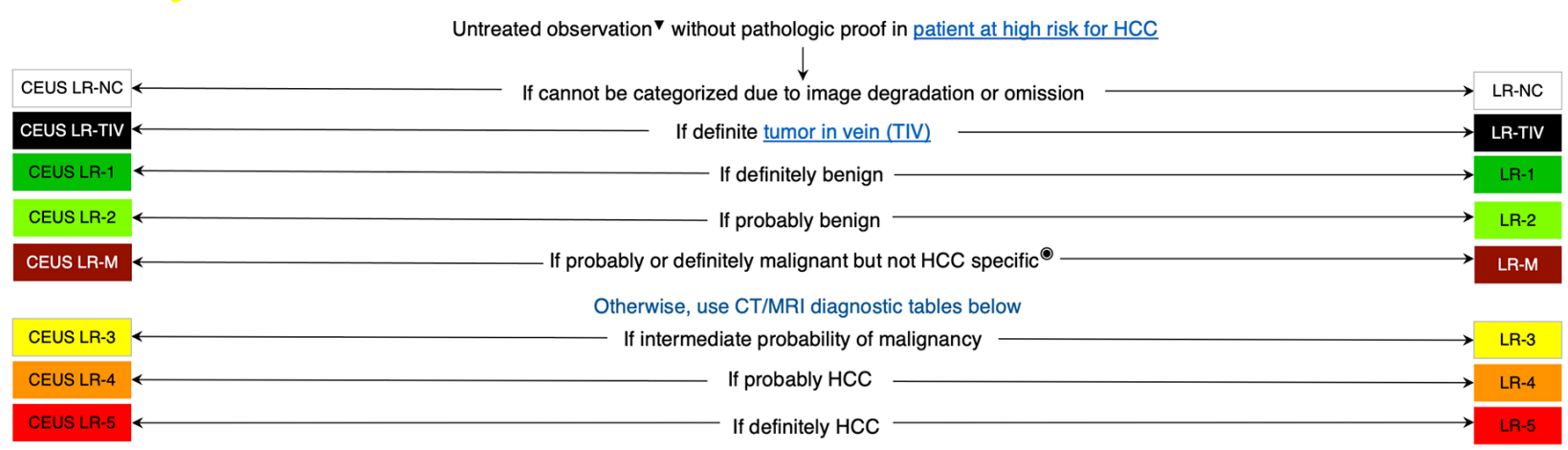

CEUS Diagnostic Table

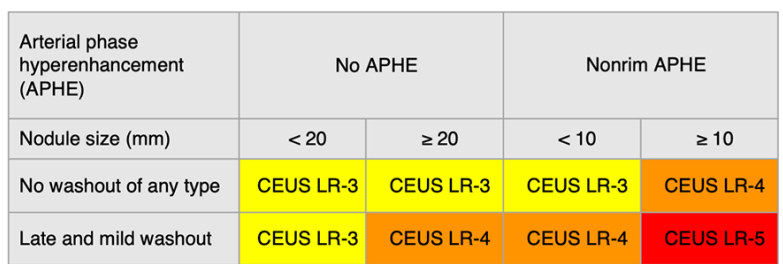

₹ For CEUS LI-RADS: Visible on pre-contrast US

O CEUS LR-M criteria - any of $\quad$ rim APHE OR following:

early $(<60 \mathrm{~s}$ ) washout OR

marked washout
CT/MRI Diagnostic Table

\begin{tabular}{|c|c|c|c|c|c|c|}
\hline \multirow{2}{*}{\multicolumn{2}{|c|}{$\begin{array}{l}\begin{array}{l}\text { Arterial phase hyperenhancement } \\
\text { (APHE) }\end{array} \\
\text { Observation size }(\mathrm{mm}) \\
\end{array}$}} & \multicolumn{2}{|c|}{ No APHE } & \multicolumn{3}{|c|}{ Nonrim APHE } \\
\hline & & \multirow{2}{*}{$\begin{array}{l}<20 \\
\text { LR-3 }\end{array}$} & \multirow{2}{*}{$\begin{array}{l}\geq 20 \\
\text { LR-3 }\end{array}$} & \multirow{2}{*}{$\begin{array}{l}<10 \\
\text { LR-3 }\end{array}$} & \multirow{2}{*}{$\begin{array}{c}10-19 \\
\text { LR-3 }\end{array}$} & \multirow{2}{*}{$\begin{array}{l}\geq 20 \\
\text { LR-4 }\end{array}$} \\
\hline $\begin{array}{l}\text { Count additional major } \\
\text { features: }\end{array}$ & None & & & & & \\
\hline \multirow{2}{*}{$\begin{array}{l}\text { - Enhancing "capsule" } \\
\text { - Nonperipheral "washout" } \\
\text { - Threshold growth }\end{array}$} & One & LR-3 & LR-4 & LR-4 & ${ }_{\text {LR-4 }}^{\text {LR-5 }}$ & LR-5 \\
\hline & $\geq$ Two & LR-4 & LR-4 & LR-4 & LR-5 & LR-5 \\
\hline
\end{tabular}

Observations in this cell are categorized based on one additional major feature: - LR-4 - if enhancing "capsule"

- LR-5 - if nonperipheral "washout" OR threshold growth

- CT/MRI LR-M criteria: Masses with targetoid or infiltrative appearance, extensive necrosis or features that in radiologist's judgment suggests non-HCC malignancy

Figure 2 LI-RADS CEUS and CT/MRI diagnostic algorithm and tables [adapted from (20)].

is performed intermittently (every 30-60 seconds) to assess late washout. Due to its high temporal capability, CEUS may provide higher sensitivity to hypervascularization and for the characterization of washout than CT or MRI $(23,25)$. Other advantages of CEUS over MRI and CT include the virtual absence of allergic reactions or nephrotoxicity of the contrast agent and lack of ionizing radiation.

CEUS LI-RADS provides a lexicon of CEUS imaging findings and reporting recommendations. Radiologists are encouraged to report the adequacy of the CEUS examination along with the timing of the acquired phases. In CEUS, the LR-NC category is usually associated with limitations of the technique, patient body habitus, or size of the observation (26). For assessing vascular occlusions, CEUS has been shown to provide high accuracy for differentiating between bland thrombus and tumor venous invasion $(27,28)$, although no studies to date have assessed CEUS LR-TIV specifically. CEUS LR-M has high sensitivity for the diagnosis of malignancy other than HCC, and therefore, may have an important role as a problemsolving tool whenever indeterminate nodules on CT or
MRI are not suitable for biopsy $(23,29)$. Despite the high sensitivity, an anticipated lower specificity of this category is observed as some HCCs with atypical imaging findings are included in this category. Interestingly, in at least one study, when HCC were mistakenly categorized as CEUS LRM, tumors tended to be poorly differentiated (30). Future studies should be encouraged to assess if imaging assigned CEUS categories may provide prognostic information on tumor biologic behavior and outcomes. The reported prevalence of HCC in the CEUS LR-3 and CEUS LR-4 categories vary. In two recent studies, the prevalence was $5 \%$ and $47 \%$ for CEUS LR-3 and $48 \%$ and $86 \%$ for CEUS LR-4 (23,30). Larger prospective studies are needed to clarify the reason behind these wide variations and to better estimate the prevalence of $\mathrm{HCC}$ in each of these categories. CEUS LR-5 has high specificity for the diagnosis of HCC (>97\%), with up to $98 \%$ of HCCs categorized as CEUS LR-5, and PPVs ranging from $97.5 \%$ to $100 \%$ depending on observation size $(23,30)$. While Korean and Chinese guidelines also endorse CEUS for the non-invasive definite diagnosis of HCC with similar criteria as LI-RADS $(6,7)$, 
Table 2 From Van der pol et al. Prevalence of overall malignancy and HCC per LI-RADS category. No HCC or overall malignancy were reported in the LR-1 category. Numbers in parentheses are confidence intervals (33)

\begin{tabular}{lcc}
\hline LI-RADS category & Rate of overall malignancy & Prevalence of HCC \\
\hline LR-2 & $3 \%(8-22 \%)$ & $14 \%(9-21 \%)$ \\
LR-3 & $40 \%(31-50 \%)$ & $38 \%(31-45 \%)$ \\
LR-4 & $80 \%(75-85 \%)$ & $74 \%(67-80 \%)$ \\
LR-5 & $97 \%(95-99 \%)$ & $94 \%(92-96 \%)$ \\
LR-M & $93 \%(87-97 \%)$ & $36 \%(26-48 \%)$ \\
LR-TIV & $92 \%(77-98 \%)$ & $79 \%(63-89 \%)$ \\
\hline
\end{tabular}

HCC, hepatocellular carcinoma.

Table 3 Performance parameters of LR-5 for the diagnosis of HCC according to imaging modality. Numbers are percentages. Numbers in parentheses are confidence intervals $(9,10,12)$

\begin{tabular}{lcccc}
\hline Imaging modality & Sensitivity $(\%)$ & Specificity (\%) & PPV (\%) & NPV (\%) \\
\hline CT & $53.7(41.3,66.1)$ & $97.3(92.2,100.0)$ & $98.7(96.3,100.0)$ & $39.5(25.5,53.5)$ \\
MRI (ECA) & $80.8(73.3,86.7)$ & $90.1(80.2,95.6)$ & $94.4(88.4,97.5)$ & $69.6(59.0,78.5)$ \\
EOB-MRI & $81.0(75.1,85.6)$ & $91.0(85.9,94.6)$ & $92.0(87.6,94.6)$ & $79.0(74.4,83.2)$ \\
\hline
\end{tabular}

$\mathrm{MRI}$, magnetic resonance imaging; HCC, hepatocellular carcinoma.

the APASL guidelines do not endorse CEUS and the Japanese guidelines advocate the use of Sonazoid which is not yet incorporated in LI-RADS $(3,5)$.

\section{CT/MRI LI-RADS}

Multiphase dynamic post-contrast CT or MRI with extracellular or intracellular contrast agents are widely considered reference standard imaging modalities for the definite diagnosis of HCC (8). LI-RADS specifies the technical standards required for diagnosis. Similar to most guidelines for the diagnosis of HCC, contrast-enhanced multiphasic dynamic imaging is mandatory. For CT examinations, multidetector scanners ( $\geq 8$ detectors) is a requisite, whereas for MRI, 1.5 or 3 Tesla magnets is required. While, CEUS LI-RADS is a relatively new algorithm, CT/MRI LI-RADS has been gaining worldwide acceptance for longer and is now one of the most used systems for the imaging diagnosis of HCC (31). In 2018, it was incorporated by the American Association for the Study of Liver Diseases (AASLD) into the North American practice guidelines for HCC (32). The wide use of LI-RADS in clinical and research settings has led to an increasing number of publications examining accuracy, advantages and gaps in knowledge related to LI-
RADS. In a recent systematic review, van der Pol et al. examined the prevalence of HCC and malignancies in general within each LI-RADS category at CT and MRI (33). For lesions categorized as LR-5, 97\% were malignant and $94 \%$ were HCC in particular. Table 2 illustrates the prevalence of malignancy and HCC per LI-RADS category among 3,556 observations included in this metanalysis.

Overall, the reported specificity of the CT/MRI LR-5 category for the diagnosis of HCC is higher than $90 \%$ $(12,34)$. For version 2018 , reported sensitivity, specificity, PPV, NPV and accuracy are $81 \%, 91 \%, 92-94.4 \%$, 69.6$79 \%$ and $83.9 \%(9,12)$. Nevertheless, small variations in performance can be seen depending on imaging modality. Some reported sensitivity, specificity, PPV, NPV values for LR-5 when LI-RADS is applied to CT, MRI with extracellular contrast and gadoxetate-enhanced MRI are shown in Table $3(9,10,12)$.

MRI is known to have slightly higher sensitivity for the diagnosis of HCC than CT, with gadoxetate disodiumenhanced MRI having the highest sensitivity, especially for observations $<20 \mathrm{~mm}(12,35)$. Currently, some Asian societies recommend gadoxetate disodium-enhanced MRI over MRI with extracellular contrast agents or CT for the diagnosis of HCC $(5,6)$. Despite the differences in accuracy 


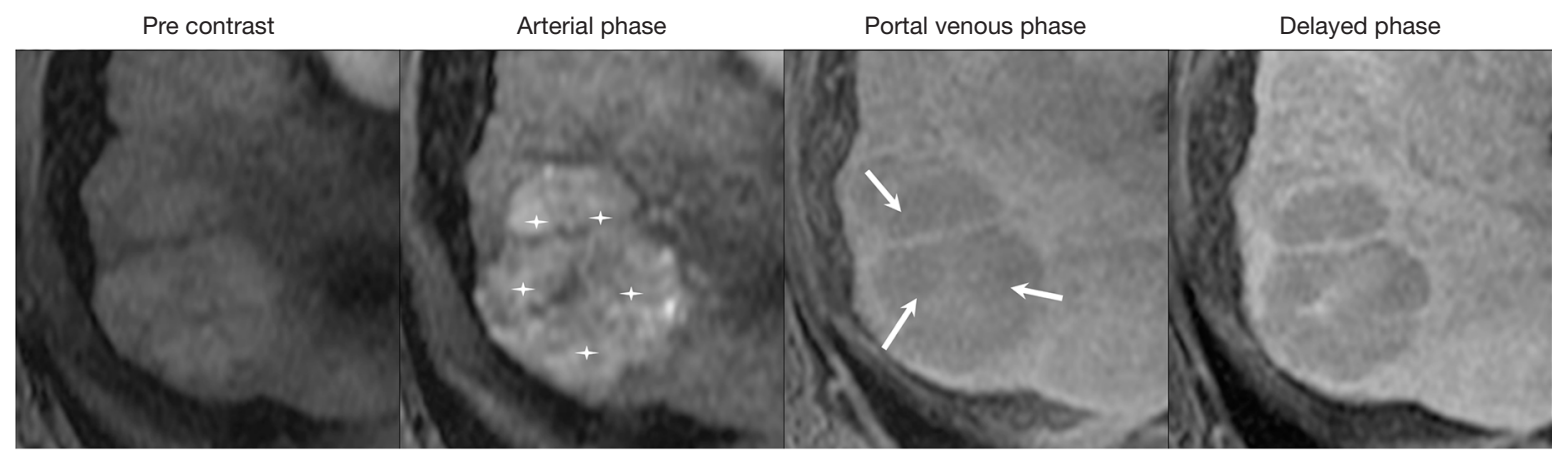

Figure 3 Gadobenate enhanced MRI: 62-year-old female with chronic hepatitis C viral infection. A 45-mm mass is seen in the right lobe with major features of HCC: APHE (stars) and washout (arrows), indicating LR-5 (definitely HCC). MRI, magnetic resonance imaging; HCC, hepatocellular carcinoma.

T1w OP

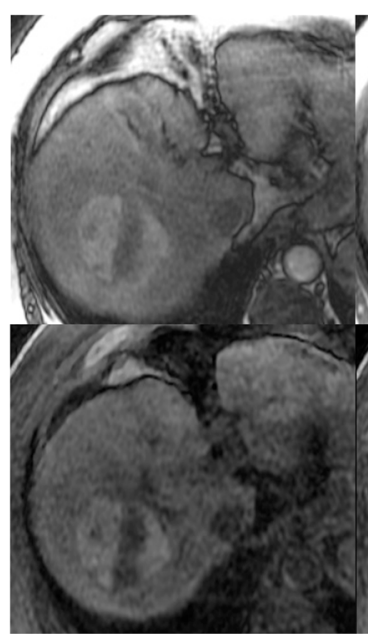

Pre contrast
T1w IP

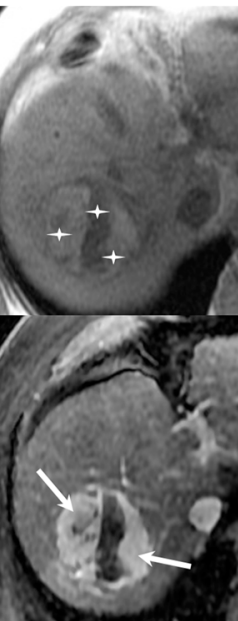

Arterial phase
T2

DWI $b=0$

$\mathrm{DW} b=750$

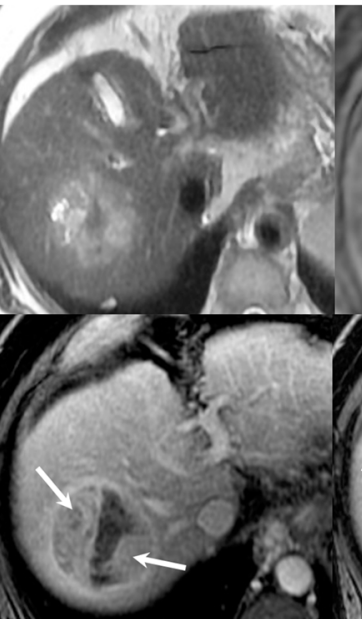

Portal Venous phase

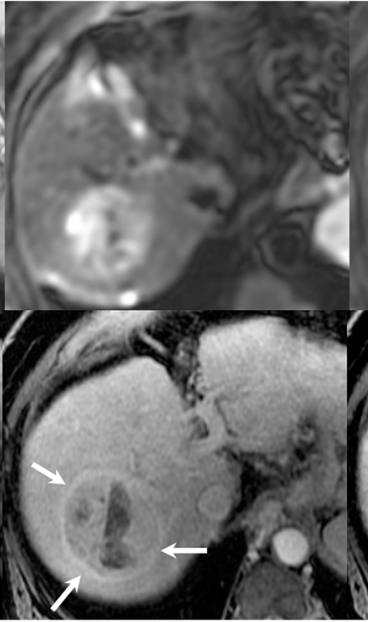

Transitional phase

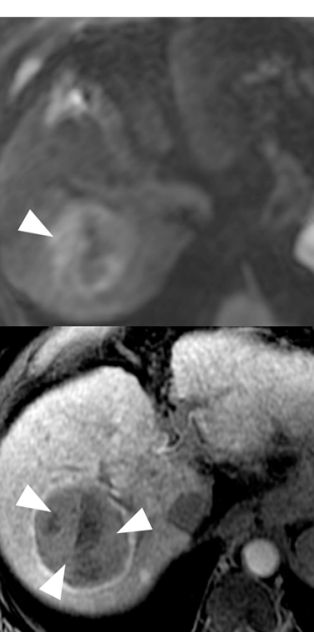

Hepatobiliary phase

Figure 4 Gadoxetate disodium enhanced MRI: 67-year-old male with chronic hepatitis $\mathrm{C}$ viral infection. A 50-mm mass in the right lobe exhibits major features of HCC (APHE, enhancing capsule and washout, arrowheads) indicating LR-5 (definitely HCC). Ancillary features favoring malignancy in general (restricted diffusion, HBP hypointensity, arrowheads) and HCC in particular (stars: mosaic architecture) are also present, although these did not affect final categorization. HCC, hepatocellular carcinoma.

described in the literature, LI-RADS does not recommend one method over another, recognizing that the choice of modality may depend on patient, institutional, and other factors. Figures 3 and 4 show major features for the diagnosis of HCC and examples of ancillary features.

Some specific clinical settings may guide the selection of one method over another. For example, the presence of liver iron overload may cause significant parenchymal signal loss on MR images and impair the assessment of relevant imaging features for lesion characterization. CT is relatively insensitive to iron deposition and therefore may be preferred in this clinical setting. Conversely, in patients with severe liver steatosis, the liver appears abnormally dark on CT images which may cause observations without fat to appear relatively hyperdense, making it difficult to assess the presence of APHE and washout. MRI with in-phase echo times and subtraction images may be preferred for improved lesion characterization. Also, in patients with impaired liver function, the hepatocellular uptake of hepatobiliary agents (HBAs) may be reduced and the hepatobiliary phase (HBP) suboptimal. This can negatively affect lesion sensitivity and adequate lesion characterization (36), and hence, in patient 
with significant liver dysfunction, the use of MRI with ECA or CT may be recommended.

The choice of extracellular versus hepatobiliary agents remains a worldwide point of controversy. For some societal guidelines, such as the APASL, Japanese and Chinese guidelines, MRI with HBAs are preferred for HCC diagnosis $(3,6)$. In v2018 CT/MRI LI-RADS only features of the extracellular phases are specific enough for the diagnosis HCC and HBP features (transitional and HBP hypointensity) are considered ancillary. The rationale behind this LI-RADS stance is explained here. In patients with preserved liver function, noticeable HBA uptake can begin immediately after the portal venous phase (PVP) (37). The resultant rapid increase in signal intensity of the liver may mistakenly cause the perception of de-enhancement of observations that are not hepatocellular in nature. Studies have shown that the characterization of washout in phases later than the PVP reduces the specificity and PPV for HCC when using gadoxetate disodium (38). To maintain high specificity, LI-RADS states that washout can only be assessed in the PVP and not during the transitional (later) phases. Likewise, HBP hypointensity is an ancillary feature of malignancy in general; any observation lacking hepatocytes could appear hypointense. This stance is consistent with the LI-RADS philosophy of striving for near perfect specificity and is a major point of difference between LI-RADS and most Asian guidelines.

Regardless of which modality and contrast agent is used, radiologists should follow the stepwise LI-RADS diagnostic algorithm.

\section{LI-RADS management recommendations}

Although LI-RADS provides tailored management recommendations for each individual category, these are considered informative guidance and should not be applied dogmatically. Management decisions in patients at risk for HCC should consider local or geographical factors, such as etiology of liver disease, population, health care resources and available treatment options, and ideally be supported by multidisciplinary discussions. For negative studies (i.e., no observations) LI-RADS recommendations are slightly different for CT/MRI and CEUS. While the former recommends return to routine surveillance, the latter considers the clinical context. If CEUS was performed due to a positive screening or surveillance US, return to surveillance is indicated. However, if CEUS was performed to better characterize an observation seen on CT/MRI, alternative imaging with CT or MRI is recommended. In both algorithms when observations are deemed non categorizable (LR-NC) due to severe image degradation or phase omission, repeat or alternative imaging within 3 months is recommended. When observations are likely benign (LR-1 and LR-2), return to routine surveillance with US is recommended. For observations at intermediate probability of malignancy (LR-3) either on CT/MRI or CEUS, LI-RADS recommends close follow-up at 3-6 months with repeat or alternative imaging, although multidisciplinary supported decision is recommended for CEUS LR-3. These include hypovascular or small $(<10 \mathrm{~mm})$ hypervascular observations.

In Asian guidelines the pursuit of a definite diagnosis through biopsy for observations $<10 \mathrm{~mm}$ is recommended (3-6). In the United States, patients may not be eligible for curative therapies (e.g., liver transplantation) until tumors reach $>20 \mathrm{~mm}$ and therefore, follow-up instead of biopsy is recommended. LR-4 observations are probable HCCs and the recommendation is for individualized multidisciplinarysupported workup that may include biopsy, presumptive treatment or follow-up. LR-5 are definite HCC and staging and treatment planning should follow without need for histological confirmation. The management of LR-M is similar, but not identical between CEUS LI-RADS and CT/MRI LI-RADS. Both support additional diagnostic work-up but while CT/MRI supports multidisciplinary discussion and biopsy consideration, CEUS LI-RADS also suggests alternative imaging. Finally, both algorithms recommend multidisciplinary discussion, biopsy and/or treatment planning for TIV observations.

\section{LI-RADS treatment response algorithm}

The LI-RADS treatment response algorithm guides the imaging assessment of response to locoregional therapy. In a similar approach to the diagnostic algorithm, the LRTR algorithm assigns categories to individual treated observations based on the presence or absence of imaging features of viability. The LR-TR Viable category is assigned to observations that after locoregional therapy exhibit nodular, masslike or irregular tissue with residual arterial phase hyperenhancement or washout or an enhancement pattern similar to the pre-treatment imaging. If the observation shows no enhancement or if only a characteristic post-treatment enhancement is seen, the LRTR Nonviable category is assigned. However, sometimes the differentiation between viable residual tumor and 
expected post-treatment enhancement is difficult. In these challenging scenarios, an intermediate category (LRTR Equivocal) is assigned. One study has found that after bland arterial embolization the rates of histologic viability in observations categorized as LR-TR Equivocal was $71 \%(39)$. For patients treated with tumor ablation (percutaneous, laparoscopic or surgical), another study has shown that more than $80 \%$ of lesions categorized as LRTR Equivocal showed signs of viable residual tumor on histopathology (40). In fact, the LR-TR Equivocal category aims to communicate a limited confidence in the diagnosis of complete response and may alert the referring physician to consider a closer follow-up or the need for retreatment.

While other treatment response algorithms (e.g., EASL, mRECIST) for HCC are intended to assess response at the patient level, LI-RADS provides criteria for response assessment on a per-lesion level. The latter is advantageous in the setting of HCC as multiple treatments are often required and the referring physician can best manage the patient to achieve disease control or downstaging. In addition to assigning TR categories, LI-RADS recommends that radiologists report the residual tumor size and provides guidance on how to perform size measurements. While the LR-TR algorithm provides for a standardize approach to the diagnosis of residual tumors in patients treated with locoregional therapies, further research should be encouraged to refine the algorithm, reduce the rate of LRTR Equivocal categorizations, and to provide clinically meaningful assessment of residual disease.

Current Asian guidelines either do not provide imaging criteria for assessment of response to treatment or defer to other imaging systems (e.g., mRECIST). The use of the LI-RADS TR algorithm could potentially provide a unified approach for clinical practice.

\section{Conclusion}

Among multiple clinical guidelines for the diagnosis and management of HCC, LI-RADS is the most comprehensive system. Four LI-RADS algorithms cover three individual clinical contexts from screening and surveillance, diagnosis and staging, and assessment of treatment response. The CEUS and CT/MRI LI-RADS algorithms provide high specificity and PPV for the noninvasive diagnosis of HCC while providing tailored management recommendations for observations found on imaging in patients at risk. The standardization of all aspects of HCC imaging rendered by LI-RADS improves the communication among healthcare professionals involved in the care of patients with or at risk for HCC, as well as the translation of research results into clinical practice. In this review we highlighted the most relevant aspects of LI-RADS for the diagnosis of HCC in clinical practice and discussed areas where LI-RADS and Asian guidelines are different. Although LI-RADS is designed for universal adoption, geographic variances in disease epidemiology and resources should be taken into consideration when applying diagnostic algorithms.

\section{Acknowledgments}

Funding: None.

\section{Footnote}

Provenance and Peer Review: This article was commissioned by the Guest Editors (Mehmet Akce and Shishir K. Maithel) for the series "Hepatocellular Carcinoma" published in Chinese Clinical Oncology. The article was sent for external peer review organized by the Guest Editors and the editorial office.

Conflicts of Interest: All authors have completed the ICMJE uniform disclosure form (available at http://dx.doi. org/10.21037/cco-20-107). The series "Hepatocellular Carcinoma" was commissioned by the editorial office without any funding or sponsorship. CBS reports grants from GE, grants from Siemens, grants from Philips, grants from Bayer, grants from Foundation of NIH, grants from Gilead, personal fees from Blade, personal fees from Boehringer, personal fees from Epigenomics, other from AMRA, other from BMS, other from Exact Sciences, other from GE Digital, other from IBM-Watson, other from Enanta, Gilead, ICON, Intercept, Nusirt, Shire, Synageva, Takeda, personal fees from Wolters Kluwer, outside the submitted work. KJF reports grants from GE, grants from Bayer, other from Bayer, other from Medscape, other from epigenomics, outside the submitted work. The authors have no other conflicts of interest to declare.

Ethical Statement: The authors are accountable for all aspects of the work in ensuring that questions related to the accuracy or integrity of any part of the work are appropriately investigated and resolved.

Open Access Statement: This is an Open Access article distributed in accordance with the Creative Commons 
Attribution-NonCommercial-NoDerivs 4.0 International License (CC BY-NC-ND 4.0), which permits the noncommercial replication and distribution of the article with the strict proviso that no changes or edits are made and the original work is properly cited (including links to both the formal publication through the relevant DOI and the license). See: https://creativecommons.org/licenses/by-nc-nd/4.0/.

\section{References}

1. Available online: http://gco.iarc.fr/ (Accessed in January 15, 2020)

2. Park JW, Chen M, Colombo M, et al. Global patterns of hepatocellular carcinoma management from diagnosis to death: the BRIDGE Study. Liver Int 2015;35:2155-66.

3. Omata M, Cheng AL, Kokudo N, et al. Asia-Pacific clinical practice guidelines on the management of hepatocellular carcinoma: a 2017 update. Hepatol Int 2017;11:317-70.

4. Yu SJ. A concise review of updated guidelines regarding the management of hepatocellular carcinoma around the world: 2010-2016. Clin Mol Hepatol 2016;22:7.

5. Kokudo N, Takemura N, Hasegawa K, et al. Clinical practice guidelines for hepatocellular carcinoma: The Japan Society of Hepatology 2017 (4th JSH-HCC guidelines) 2019 update. Hepatol Res 2019;49:1109-13.

6. Xie DY, Ren ZG, Zhou J, et al. Critical appraisal of Chinese 2017 guideline on the management of hepatocellular carcinoma. Hepatobiliary Surg Nutr 2017;6:387.

7. Korean Liver Cancer Association (KLCA); National Cancer Center (NCC), Goyang, Korea. 2018 Korean Liver Cancer Association-National Cancer Center Korea Practice Guidelines for the Management of Hepatocellular Carcinoma. Korean J Radiol 2019;20:1042-113.

8. Tang A, Cruite I, Mitchell DG, et al. Hepatocellular carcinoma imaging systems: why they exist, how they have evolved, and how they differ. Abdom Radiol (NY) 2018;43:3-12.

9. Lee SM, Lee JM, Ahn SJ, et al. LI-RADS Version 2017 versus Version 2018: Diagnosis of Hepatocellular Carcinoma on Gadoxetate Disodium-enhanced MRI. Radiology 2019;292:655-63.

10. Alhasan A, Cerny M, Olivié D, et al. LI-RADS for CT diagnosis of hepatocellular carcinoma: performance of major and ancillary features. Abdom Radiol (NY) 2019;44:517-28.

11. Fowler KJ, Tang A, Santillan C, et al. Interreader reliability of LI-RADS version 2014 algorithm and imaging features for diagnosis of hepatocellular carcinoma: a large international multireader study. Radiology 2018;286:173-85.

12. Ren AH, Zhao PF, Yang DW, et al. Diagnostic performance of MR for hepatocellular carcinoma based on LI-RADS v2018, compared with v2017. J Magn Reson Imaging 2019;50:746-55.

13. Lim K, Kwon H, Cho J. Inter-reader agreement and imaging-pathology correlation of the LI-RADS M on gadoxetic acid-enhanced magnetic resonance imaging: efforts to improve diagnostic performance. Abdom Radiol (NY) 2020;45:2430-9.

14. Zhang YD, Zhu FP, Xu X, et al. Classifying CT/MR findings in patients with suspicion of hepatocellular carcinoma: Comparison of liver imaging reporting and data system and criteria-free Likert scale reporting models. J Magn Reson Imaging 2016;43:373-83.

15. Masuzaki R, Tateishi R, Yoshida H, et al. Prospective risk assessment for hepatocellular carcinoma development in patients with chronic hepatitis $\mathrm{C}$ by transient elastography. Hepatology 2009;49:1954-61.

16. Shin SH, Kim SU, Park JY, et al. Liver stiffness-based model for prediction of hepatocellular carcinoma in chronic hepatitis B virus infection: comparison with histological fibrosis. Liver Int 2015;35:1054-62.

17. Jung KS, Kim SU, Ahn SH, et al. Risk assessment of hepatitis B virus-related hepatocellular carcinoma development using liver stiffness measurement (FibroScan). Hepatology 2011;53:885-94.

18. Li J, Zou B, Yeo YH, et al. Prevalence, incidence, and outcome of non-alcoholic fatty liver disease in Asia, 1999-2019: a systematic review and meta-analysis. Lancet Gastroenterol Hepatol 2019;4:389-98.

19. Piscaglia F, Svegliati-Baroni G, HCC-NAFLD Italian Study Group. Clinical patterns of hepatocellular carcinoma in nonalcoholic fatty liver disease: A multicenter prospective study. Hepatology 2016;63:827-38.

20. Available online: https://www.acr.org/Clinical-Resources/ Reporting-and-Data-Systems/LI-RADS (accessed in February 25, 2020)

21. Tang A, Bashir MR, Corwin MT, et al. Evidence supporting LI-RADS major features for CT-and MR imaging-based diagnosis of hepatocellular carcinoma: a systematic review. Radiology 2018;286:29-48.

22. Jang HJ, Kim TK, Burns PN, et al. CEUS: An essential component in a multimodality approach to small nodules in patients at high-risk for hepatocellular carcinoma. Eur J 
Radiol 2015;84:1623-35.

23. Terzi E, Iavarone M, Pompili M, et al. Contrast ultrasound LI-RADS LR-5 identifies hepatocellular carcinoma in cirrhosis in a multicenter restropective study of 1,006 nodules. J Hepatol 2018;68:485-92.

24. Lyshchik A, Kono Y, Dietrich CF, et al. Contrastenhanced ultrasound of the liver: technical and lexicon recommendations from the ACR CEUS LI-RADS working group. Abdom Radiol (NY) 2018;43:861-79.

25. Hu J, Bhayana D, Burak KW, et al. Resolution of indeterminate MRI with CEUS in patients at high risk for hepatocellular carcinoma. Abdom Radiol (NY) 2020;45:123-33.

26. Wilson SR, Lyshchik A, Piscaglia F, et al. CEUS LIRADS: algorithm, implementation, and key differences from CT/MRI. Abdom Radiol (NY) 2018;43:127-42.

27. Tarantino L, Francica G, Sordelli I, et al. Diagnosis of benign and malignant portal vein thrombosis in cirrhotic patients with hepatocellular carcinoma: color Doppler US, contrast-enhanced US, and fine-needle biopsy. Abdom Imaging 2006;31:537-44.

28. Sorrentino P, D'Angelo S, Tarantino L, et al. Contrastenhanced sonography versus biopsy for the differential diagnosis of thrombosis in hepatocellular carcinoma patients. World J Gastroenterol 2009;15:2245.

29. Tan Z, Teoh WC, Wong KM, et al. Analysis of comparative performance of CEUS and CECT/MR LIRADS classification: Can CEUS dichotomize LI-RADS indeterminate lesions on CT or MRI? Clin Imaging 2020;62:63-8.

30. Huang JY, Li JW, Lu Q, et al. Diagnostic Accuracy of CEUS LI-RADS for the Characterization of Liver Nodules $20 \mathrm{~mm}$ or Smaller in Patients at Risk for Hepatocellular Carcinoma. Radiology 2020;294:329-39.

31. Tang A, Abukasm K, Song B, et al. Imaging of hepatocellular carcinoma: A preliminary International Survey. Abstract presentation - Radiological Society of North America (RSNA) 104th Scientific Assembly and Annual Meeting, November 2018, Chicago, USA.

Cite this article as: Cunha GM, Sirlin CB, Fowler KJ. Imaging diagnosis of hepatocellular carcinoma: LI-RADS. Chin Clin Oncol 2021;10(1):3. doi: 10.21037/cco-20-107
32. Heimbach JK, Kulik LM, Finn RS, et al. AASLD guidelines for the treatment of hepatocellular carcinoma. Hepatology 2018;67:358-80.

33. van der Pol CB, Lim CS, Sirlin CB, et al. Accuracy of the liver imaging reporting and data system in computed tomography and magnetic resonance image analysis of hepatocellular carcinoma or overall malignancy-a systematic review. Gastroenterology 2019;156:976-86.

34. Kierans AS, Makkar J, Guniganti P, et al. Validation of Liver Imaging Reporting and Data System 2017 (LIRADS) Criteria for Imaging Diagnosis of Hepatocellular Carcinoma. J Magn Reson Imaging 2019;49:e205-15.

35. Semaan S, Violi NV, Lewis S, et al. Hepatocellular carcinoma detection in liver cirrhosis: diagnostic performance of contrast-enhanced CT vs. MRI with extracellular contrast vs. gadoxetic acid. Eur Radiol 2020;30:1020-30.

36. Kim JY, Lee SS, Byun JH, et al. Biologic factors affecting HCC conspicuity in hepatobiliary phase imaging with liver-specific contrast agents. AJR Am J Roentgenol 2013;201:322-31.

37. Chernyak V, Fowler KJ, Heiken JP, et al. Use of gadoxetate disodium in patients with chronic liver disease and its implications for liver imaging reporting and data system (LI-RADS). J Magn Reson Imaging 2019;49:1236-52.

38. Joo I, Lee JM, Lee DH, et al. Retrospective validation of a new diagnostic criterion for hepatocellular carcinoma on gadoxetic acid-enhanced MRI: can hypointensity on the hepatobiliary phase be used as an alternative to washout with the aid of ancillary features? Eur Radiol 2019;29:1724-32.

39. Shropshire EL, Chaudhry M, Miller CM, et al. LIRADS Treatment Response algorithm: performance and diagnostic accuracy. Radiology 2019;292:226-34.

40. Chaudhry M, McGinty KA, Mervak B, et al. The LIRADS version 2018 MRI treatment response algorithm: evaluation of ablated hepatocellular carcinoma. Radiology 2020;294:320-6. 